\title{
Corrupción, inversión pública y crecimiento ${ }^{1}$
}

Vito Tanzi y H amid Davoodi *

\section{Introducción}

H asta que un gran escándalo de corrupción, popularmente denominado tangentopoli (ciudad soborno), derribó la clase política que había gobernado Italia durante varias décadas, ese país había dado cuenta de una de las mayores cuotas de gasto de capital en el PIB entre los países de la OCDE. D espués de que estalló el escándalo y de que varios individuos de relieve fueron a la cárcel e incluso se suicidaron, el gasto de capital cayó bruscamente. La caída parece haber sido causada por una reducción del núme ro de proyectos de importancia que se iban a acometer y quizá, lo que es más importante, por una brusca caída en los costes de los proyectos que todavía se estaban llevando a cabo. La información facilitada por Transparencia Internacional (TI) ${ }^{2}$ señala que, en un período de dos o tres años, en la ciudad de M ilán, la ciudad donde estalló el escándalo en primer lugar, el coste de las conexiones de los ráles de la ciudad cayó aproximadamente en un $52 \%$, el coste de un kilómetro de metro cayó alrededor de un $57 \%$ y el presupuesto para la nueva terminal del aeropuerto se redujo en un 59\% como reflejo de la reducción de los costes de construcción. Aunque hay que ser consciente de la falacia lógica de post hoc, ergo proter hoc, la conexión entre los dos acontecimientos es demasiado fuerte para considerarse una coincidencia. De hecho, este documento adopta la perspectiva de que no podría haber sido una coincidencia.

La hipótesis básica de este trabajo es que la corrupción, y especialmente la corrupción política o corrupción «a gran escala» ${ }^{3}$, está unida con frecuencia a los proyectos importantes. Es probable que la corrupción incremente el número de proyectos que se llevan a cabo en un pás, y que cambie el diseño de estos proyectos aumentando su tamaño y complejidad. El resultado neto es: (a) un incremento en la cuota de la inversión pública en el PIB; (b) una caída en la productividad media de esa inversión y a causa de otras restricciones presupuestarias y otras consideraciones, (c) una posible reducción en otras categorías del gasto público, tales como «funcionamiento y mantenimiento», educación y sanidad. Como consecuencia de este y otros efectos de la corrupción sobre la economía, la tasa de crecimiento de un país en donde la corrupción es significativa se ve afectada negativamente.

En la sección II comentamos las razones por las que suponemos que la inversión pública es particularmente sensible a la existencia de la corrupción (política). En la sección III presentamos los datos empíricos sobre las hipótesis básicas. En la sección IV sacamos conclusiones.

\section{Corrupción y gasto de Gobierno}

Al menos desde después de la II Guerra M undial, cuando los economistas influyentes como H arrod, D omar, Rostow y otros argumentaron que los páses necesitaban capital para crecer y lo que es más importante, que existe una relación casi mecánica (el ratio de capital-output) entre el incremento del gasto de capital y el incremento del crecimiento, ha habido un fuerte sesgo intelectual en la profesión económica a favor del gasto de capital. Por ejemplo, cuando los economistas evalúan la distribución de dinero público entre el gasto corriente y gasto de capital en los presupuestos de los gobiernos, tienden a criticar a los países que permiten que crezca la cuota de gasto corriente. Por otra parte, 
generalmente elogian a los países cuya cuota de gasto de capital en el total de los gastos del gobierno aumenta.

El sesgo anterior seresumeen la «regladeoro»quemuchoseconomistas recomiendan a los países. Esa regla establece en esencia que es correcto pedir prestado en tanto en cuanto el préstamo se destinea proyectos deinversión ${ }^{4}$. Por tanto, es correcto pedir prestado para financiar la construcción de nuevas carreteras, pero no para financiar la reparación de las carreterasya existentes; o pedir prestado parala construcción de un nuevo hospital, pero no para contratar doctores o enfermeras o para comprar medicinas. Esta regla se sigue invocando como una buena guía de las políticas públicas, incluso ante muchos datosqueevidencian que realizar algunos gastoscorrientes - como «funcionamiento y mantenimiento», que mantienen las infraestructuras existentes en buenas condiciones, o el gasto que contribuye a la acumulación del capital humano- puede promover el crecimiento, más queel gasto de capital.

Los políticos han internalizado este sesgo y hasta cierto punto lo han explotado. Por ejemplo, las ceremonias de cortar la cinta, cuando se terminan e inauguran los nuevos proyectos de inversión relativos a carreteras, presas, canales de riego, centrales eléctricas, puertos, aeropuertos, escuelas y hospitales, les hacen muy popularesa los políticos. Les gusta versefotografiados en los artículos de los periódicos en el acto de cortar la cinta y, por tanto, cabe presumir que contribuyendo al crecimiento futuro del país. En un país latinoamericano concreto, los proyectos importantes terminados bajo la actual administración se han pintado de naranja para enviar a la población una clara señal de que el gobierno actual está promoviendo el crecimiento. Este sesgo pro-inversión incrementa el presupuesto en inversión. Sostendremos que otro factor que también incrementa la dimensión del presupuesto en inversión es la corrupción.

No hay nada rutinario en el presupuesto en inversión y su composición. M ientras una parte importante del gasto actual del gobierno refleja, en gran medida, derechos explícitos o implícitos o compromisos previos ${ }^{5}$, lo cual otorga, por tanto, un poder discrecional a corto plazo a los políticos y especialmente a algunos políticos en concreto, el gasto de capital es altamente discrecional ${ }^{6}$. En cuanto a este último, las grandes figuras políticas - miembros del Parlamento, secretarios generales, ministros e incluso jefes de Estado- deben tomar algunas de las decisiones básicas. Estas decisiones se refieren a: (a) la dimensión del presupuesto total en inversión pública; (b) la composición general de ese presupuesto, p.e., la distribución en grandes líneas entre las distintas categorías de gasto de capital; (c) la elección de proyectos específicos y su ubicación; y (d) incluso el tamaño y el diseño de cada proyecto. En estas decisiones y especialmente en las de las letras (c) y (d), algunos individuos de alto nivel tendrán un control o influencia considerable. Esto ocurrirá especialmente cuando alguna de las instituciones de control 0 auditoría fundamentales no estén bien desarrolladas y, por tanto, los controles institucionales sean débiles.
Los proyectos de inversión pública tienden a ser grandes y en algunos casos, son muy grandes. Su ejecución se contrata frecuentemente con empresas privadas, nacionaleso extranjeras. Por tanto, hay una necesidad de escoger la empresa que será responsable de acometer el proyecto. Para una empresa privada, obtener un contrato para ejecutar un proyecto, y especial mente uno grande, puede ser muy beneficioso. Por tanto, los gestores de estas empresas pueden estar dispuestos a pagar una «comisión» a los funcionarios del gobierno para que les ayuden a conseguir el contrato ${ }^{7}$. En al gunos países las comisiones pagadas por sus empresas a políticos extranjeros son legales y fiscalmente deducibles. Tales «comisiones» se calculan a menudo como porcentajes del coste total de los proyectos.

Una comisión de unos pocos puntos porcentuales sobre un proyecto que cuesta millones o incluso cientos de millones de dólares puede suponer una gran suma, lo suficientemente grande como para sobrepasar el precio de la tentación de muchos individuos $^{8}$. Cuando las comisiones se calculan como un porcentaje del coste de los proyectos, los funcionarios públicos que reciben los pagos por ayudar a las empresas a conseguir el contrato tendrán un interés personal en incrementar el alcance o la dimensión de los proyectos a fin de poder obtener mayores comisiones ${ }^{9}$.

El proceso de aprobación de un proyecto de inversión consta de varias fases. Por ejemplo, un proyecto de construcción civil (carreteras, edificios, puertos) requiere de decisiones relativas a: (a) temas de especificación y diseño; (b) aspectos de la propuesta (¿limitada 0 abierta?); (c) escrutinio de la propuesta; (d) negociaciones de la propuesta; y (e) proceso de aprobación y contratación de la propuesta. La finalización del proyecto requerirá verificar que el trabajo se ha realizado según lo estipulado en el contrato. También requerirá de cierto arbitraje sobre los puntos de desacuerdo. La puesta por escrito de los contratos para proyectos complejos es muy difícil e inevitablemente habrá muchas áreas de incertidumbre y eventual desacuerdo.

En algunas de estas fases, será posible que un funcionario de alto nivel posicionado estratégicamente influya en el proceso de distintas formas que conduzcan a la elección de una empresa en concreto. Por ejemplo, las especificaciones del diseño pueden ser estar hechas a medida para una empresa dada. La realización de las propuestas puede ir acompañada de suministro de información interna para favorecer a las empresas y así sucesivamente.

La empresa que paga la comisión no sufrirá por el pago del soborno si es capaz de recuperar ese coste de diferentes maneras: (a) mediante una recuperación abierta y pública del coste, si puede superar la oferta de los competidores con una oferta que incluya el coste de la comisión; (b) puede llegar a un entendimiento con el funcionario influyente de que la baja oferta inicial se pueda ajustar al alza más adelante, presumiblemente para reflejar modificaciones en el diseño básico ${ }^{10} ; 0$ (c) reducir los costes del proyecto, escatimando la calidad del trabajo realizado y de los 
materiales empleados, entregando por tanto a la finalización, un producto de inferior calidad ${ }^{11}$. En los casos en los que el contrato se ha estipulado en base a un precio, la empresa puede recuperar el coste de la comisión aumentando el precio.

En todas estas alternativas que requieren de la colaboración del funcionario corrupto, el país acabará, bien teniendo un costemayor por el proyecto en concreto delo que habría sido en el caso de que no hubiese habido corrupción; bien con un proyecto mayor 0 más complejo de lo que habría sido necesario; o bien con un proyecto de inferior calidad que no se ha realizado de acuerdo con los estándares previstos y requerirá de mantenimiento y reparaciones muy costosas. La experiencia con los proyectos del sector público, especialmente en los países en vías de desarrollo, está llena de historias de carreteras que debían repararse en un breve período de tiempo después desu finalización, centrales eléctricas que funcionaban a una capacidad muy inferior de la prevista, etc.

El argumento anterior ha puesto de manifiesto casos en donde personalidades políticas o funcionarios de alto nivel corruptos dirigen la aprobación de proyectos de inversión hacia empresas concretas, nacionales o extranjeras, a cambio de sobornos. Esta es una parte importante de la forma en que la corrupción, definida en el sentido más amplio de búsqueda de rentas, afecta a la inversión pública. Sin embargo, aquí no acaba todo. Se producen casos importantes de corrupción también cuando las personalidades políticas dirigen la inversión pública hacia sus propios distritos o su propia tierra. En un reciente caso que apareció en el Financial Times del 29 de julio de 1997, el presidente de un país fue acusado de haber construido un aeropuerto con fondos públicos en su pequeña ciudad natal, aunque parecía haber escasa justificación económica para ello. Esto no es un caso aislado. En otras ocasiones, los proyectos se dirigen hacia lugares concretos para incrementar el valor de las posesiones (como tierras propiedad de las personalidades políticas) en dichos lugares.

En todos estos casos, se reduce la productividad del gasto de capital, reduciendo, por tanto, la tasa de crecimiento del país. Por consiguiente, la corrupción puede distorsionar significativamente la relación entre el input del capital y el output generado por ese capital, incrementando pues, el ratio capital-output.

Cuando la aprobación de los proyectos de inversión llega a estar mucho más influida por los funcionarios corruptos de alto nivel, la tasa de recuperación de la inversión de los proyectos calculada en un análisis de coste/ben eficio deja de ser el criterio para la selección de proyectos ${ }^{12}$. El gasto de capital llega a ser mucho menos productivo y a contribuir mucho menos al crecimiento de lo que generalmente se piensa. D esafortunadamente, no son extrañas situaciones de este tipo. En estas situaciones, a aquéllos que llevan a cabo los proyectos (las empresas ejecutoras) lo que más les importa son los beneficios que consiguen. $Y$ a las figuras políticas que autorizan los proyectos y eligen las empresas, lo que más les importa son los sobornos o las demás ventajas que puedan conseguir. Por tanto, la corrupción distorsiona todo el proceso de toma de decisiones relacionado con el presupuesto de la inversión. En casos extremos de países totalmente corruptos, los proyectos se escogen exclusivamente por su capacidad generadora de sobornos y no por su productividad. La productividad de los proyectos llega a ser casi irrelevante ${ }^{13}$.

Cuando la corrupción juega un papel importante en la selección de los proyectos y contratistas, el resultado de este proceso es un presupuesto de capital altamente distorsionado. Surgen los «elefantes blancos» y las «catedrales en el desierto». Algunos proyectos se terminan, pero no se usan nunca. Algunos son más grandes y complejos delo necesario. Algunos son deuna calidad tan baja que necesitarán continuas reparaciones y su productividad estará muy por debajo de las expectativas iniciales. En estas circunstancias, no essorprendente queel gasto de capital no genere los resultadosque los economistas esperan en términos de crecimiento.

La corrupción generalizada en el presupuesto de inversión no sólo reducirá la tasa de recuperación de nueva inversión pública, sino quetambién afectaráa la tasa de recuperación que un país obtiene de sus infraestructuras ya existentes. Varias son las razones.

Primero, en la medida en que la corrupción no es un nuevo fenómeno, sino que ya ha existido durante al gún tiempo, las infraestructuras existentes también se han contaminado, porque las inversiones pasadas también estuvieron mal dirigidas o distorsionadas por la corrupción.

En segundo lugar, un mayor gasto en proyectos importantes reducirálos recursos disponiblespara otros proyectos. D elos otros gastos, uno que no está protegido por la existencia de derechos o compromisos implícitos es «funcionamiento y mantenimiento», que es la clase de gasto público corriente que se necesita para mantener las infraestructuras físicas existentes de un país en buenas condiciones de funcionamiento. Por tanto, un fenómeno que se observa frecuentemente son las pobres condiciones en que se encuentran las infraestructuras existentes (carreteras con baches, edificios en malas condiciones que necesitan reparaciones, etc.). A menudo se observan situaciones en donde se acometen nuevos proyectos mientras que se deja que las estructuras existentes se deterioren.

Tercero, y más bien a modo de especulación, en los casos de corrupción extrema, se puede reducir intencionalmente el gasto en funcionamiento y mantenimiento de la infraestructura física de un país para que algunas infraestructuras, como las carreteras, se deterioren rápidamente hasta el punto de que se tengan que reconstruir, ofreciendo por tanto a los funcionarios de alto nivel la oportunidad de conseguir otra comisión de la empresa que lleve a cabo el proyecto. Algunos Informes del Banco Mundial han insinuado que esto puede haber pasado en al gunos países.

Un país puede sacar más jugo a las infraestructuras existentes, manteniéndolas en buenas condiciones de funcionamiento para que puedan usarse casi al 100\% de su capacidad ${ }^{14}$. Esfácil pensar 
en situacionesen lasqueel deterioro deestasinfraestructuras retrasa el crecimiento más delo quelosnuevosproyectosimportantesañaden a dicho crecimiento. Además, cuando la corrupción generalizada en un país reducelos recursospor el impacto negativo quetiene en los ingresos fiscales (lo cual viene producido por administradores fiscales corruptos), el gasto en funcionamiento y mantenimiento se reducirá másquelainversión pública, debido al sesgo intelectual mencionado másarriba queapoya el pedir préstamos para proyectos de importancia, pero no para gasto corriente.

\section{Análisis empírico}

\section{A. Descripción de datos}

En nuestro análisis empírico empleamos índices de datos de corrupción de dos fuentes: Negocio Internacional (Business Internacional o BI) y los Servicios de Riesgo Político (Political Risk Services, Inc.). M Auro (1995), entre otros, ha empleado el índice BI, que está disponible para 68 países en el período 1980-83 (una observación por país). La segunda fuente publica un índice íntimamente relacionado con éste en la Guía Internacional de Riesgo por Países (International Country Risk Guide ICRG). A diferencia del índice BI, el índice ICRG es anual; cubreel período de 1982-95 y, dependiendo del año, está disponible desde 42 hasta 95 países. Knack y KeEFER (1995), entre otros, han empleado este índice.

Ambosíndices son valoraciones del grado decorrupción en un país realizadas por observadores informados, la red de corresponsales de BI , en el caso del índice BI, einversores extranjeros, en el caso del índiceICRG. El índiceBI sehainterrumpido, mientras el índice ICRG se actualiza anual mentey se vende como partedeun paquetea inversores potencialesa nivel mundial . Lacorrupción en el BI indica «el grado en que las transacciones comerciales tienen que ver con la corrupción o con pagos cuestionables». El índice va de 0 (más corrupto) a 10 (el menos corrupto). En el índiceICRG la mayor corrupción indica «la probabilidad de que los altos funcionarios del gobierno demanden pagos especiales» y que «cabe esperar con carácter general pagos ilegales en todos los niveles inferiores del gobierno» en forma de «sobornos relacionados con las licencias de importación y exportación, controles de intercambio, valoraciones fiscales, protección policial o préstamos». El índice ICRG va de 0 (más corrupto) a 6 (el menos corrupto).

H emos reelaborado la escala del índice ICRG multiplicándolo por 10/6, de tal forma que ambosíndices vayan de 0 a 10 y los hemos juntado para formar un único índice de corrupción desde 1980 a $1995{ }^{15}$. Para facilitar la interpretación de los resultados de la regresión, hemos multiplicado el índice resultante por me- nos uno, para que los valores más altos del índice signifiquen una mayor corrupción.

El estudio de la sección II ha subrayado la interacción entre la corrupción, la inversión pública, los gastos en funcionamiento y mantenimiento ( $F \& M$ ) y otros aspectos de la posición presupuestaria del gobierno. Para la inversión pública, se emplean los datos sobre el gasto de capital de las Estadísticas de Finanzas del Gobierno (Government Finance Statitstics o GFS) del Fondo $M$ onetario Internacional. D esafortunadamente, no se dispone de los datos comparativos entre países sobre los gastos en $F \& M$. H emos escogido, por tanto, dos sustitutos denominados «gasto sobre otros bienes y servicios» que incluye los gastos en $F \& M$, y "suel dos y salarios como parte de los gastos corrientes». La lógica que subyace en estos sustitutos se explicará más adelante.

Para investigar el impacto de la corrupción sobre la calidad de la inversión pública, usamos los siguientes indicadores de calidad de las infraestructuras:

- Carreteras asfaltadas en buenas condiciones como porcentaje del total de las carreteras asfaltadas.

- Pérdidas en el sistema de energía eléctrica como porcentaje de la producción total de energía.

- Fallos en las telecomunicaciones por cada 100 líneas importantes por año.

- Pérdidas de agua como porcentaje del abastecimiento total de agua.

- Ferrocarriles diesel en uso como porcentaje del inventario diesel total.

A menudo, se aludea los datos anteriores como indicadores de gestión de las infraestructuras y parecen adecuados para nuestro propósito; se miden desde la perspectiva tanto de los proveedores como de los usuarios de las infraestructuras', abarcan un gran número de países y, lo que es más importante, tienen muchas características que son responsabilidad de los gobiernos. Estos datos se han tomado del Sindicato Internacional de Telecomunicaciones (International Telecommunications U nion) y de la base de datos de los Indicadores de D esarrollo M undiales (World D evelopment Indicators) del Banco M undial. Las carreteras asfaltadas en buenas condicionesson carreteras sustancialmentelibres de problemasimportantes y precisan sólo del mantenimiento de rutina. Las pérdidas del sistema de energía eléctrica consisten en pérdidas técnicas, como las pérdidas de resistencia en la transmisión y distribución y pérdidas no técnicas, como las conexionesilegales a la electricidad y otrasfuentes de robos. Laspérdidas del sistema se expresan, pues, como una fracción de la producción total. Los fallos en las telecomunicaciones por cada 100 líneas importantes por año se refieren al número de fallos comunicados por 100 líneas importantes cada año. Las pérdidas de agua incluyen pérdidas físicas (roturas de tuberías y desbordamientos) y pérdidas comerciales (registro de menos contadores de los debidos, uso ilegal que incluye las conexio- 
nesfraudulentas o no registradas, y los usos legales, pero no medidosgeneralmente, como la lucha contra incendios). Losferrocarriles diesel en uso como porcentaje del total del inventario diesel mide la gestión técnica y de dirección.

Finalmente, los datos sobre los ingresos del gobierno, tomados del GFS, se expresan como fracciones del PIB. Los datos del PIB y del PIB real per capita (este último es una variable de control en la regresión) proceden de la base de datos de los Indicadores de Desarrollo M undial (World Development Indicators) del Banco Mundial.

\section{B. Resultados de la regresión}

El estudio de la sección II sugiere unas hipótesis testeables sobre la relación entre la corrupción de una parte y la inversión pública, los ingresos del gobierno, los gastos en F\&M y la calidad de las infraestructuras, de otra. Usamos un análisis de regresión para poner a prueba estas hipótesis empleando datos comparativos entre países. D esde luego, es difícil extraer afirmaciones de causalidad de las ecuaciones de la regresión y uno se debe guardar de posibles resultados falsos de la regresión. Esto se hace controlando otras variables, tales como el PIB real per capita, el ratio ingresos del gobierno-PIB y el ratio inversión pública-PIB.

\section{1) Corrupción e inversión pública}

H ipótesis 1: Permaneciendo igual el resto de cosas, un elevado nivel de corrupción se asocia con una alta inversión pública.

Para poner a prueba esta hipótesis, hacemos una regresión del ratio de inversión pública-PIB sobre una constantey el índice de corrupción. D espués, añadimos el ratio de ingresos del gobierno y PIB real per capita para ver si la relación entre la corrupcióninversión resiste la inclusión de estas dos variables. Añadimos el PIB real per capita, puesto que es típicamente un sustituto de la etapa del desarrollo económico y los diferentes niveles de desarrollo pueden precisar diferentes necesidades de inversión pública. Se añade el ratio ingresos del gobierno-PIB, porque cuanto mayores son estos ingresos, más fácil es financiar la inversión pública. Los resultados son tres regresiones que se muestran en la Tabla 1. En todas estas regresiones, no podemos negar la hipótesis con una relevancia del $1 \%{ }^{16}$. La variable ingresos del gobierno-PIB tiene un coeficiente positivo estadísticamente significativo, que indica que tales ingresos son una fuente importante de financiación de la inversión pública. Los resultados mostrados en la Tabla 1 lo son para una muestra mundial, pero también son válidos para las submuestras de los países en vías de desarrollo y los miembros de la O rganización para la Cooperación y el D esarrollo Económico (OCDE).
Tabla 1

L os Efectos de la Corrupción en la Inversión Pública, 1980-95

(Como ratio de PIB, datos anuales)

\begin{tabular}{lccc|} 
Variables independientes & (1) & (2) & (3) \\
\hline Constante & 6,75 & 6,47 & 4,71 \\
& $(23,4)$ & $(19,5)$ & $(13,9)$ \\
& & & \\
Índice de corrupción & 0,38 & 0,27 & $0,48)$ \\
& $(8,97)$ & $(4,15)$ & $(7,48)$ \\
& & $-0,71$ & $-1,21$ \\
PIB* real per capita & & $(-2,94)$ & $(-5,18)$ \\
& & & 0,13 \\
Ratio ingresos del gobierno-PIB & & & $(12,6)$ \\
& & & 0,207 \\
& 0,069 & 0,082 & 1.000 \\
\hline R2 ajustado & 1.081 & 1.011 & \\
N úmero de observaciones & & & \\
\end{tabular}

Fuentes: FMI, Estadísticas de Finanzas del Gobierno; Tablas M undiales; Negocio Internacional; y Servicios de Riesgo Político. El índice de corrupción se ha tomado de M auro (1995) y la Guía Internacional de Riesgo por Países (International Country Risk Guide) compilada por Servicios de Riesgo Político (Political Risk Services). Un valor alto del índice significa que un país tiene un elevado nivel de corrupción; t-estadísticas están entre paréntesis. La técnica de estimación es O LS.

* Indica que el coeficiente está multiplicado por 10.000 .

\section{2) La corrupción y los ingresos del gobierno}

Las regresiones de la Tabla 1 muestran el impacto directo de la corrupción sobre la inversión pública y no excluyen la posibilidad de un impacto indirecto, digamos, a través de los ingresos del gobierno. La corrupción puede reducir los ingresos del gobierno si contribuye a la evasión fiscal, a las exenciones fiscales indebidas 0 a una débil administración fiscal. Esto nos lleva a la segunda hipótesis:

H ipótesis 2: Permaneciendo el resto de cosas igual, un elevado nivel de corrupción se asocia con unos ingresos del gobierno escasos.

Para poner a prueba estaafirmación, hacemos una regresión del ratio de ingresos del gobierno-PIB sobre una constantey sobrela corrupción. Entonces añadimosel PIB real per capita paracontrolar los efectos de la fase del desarrollo económico. Los resultados recogidos en la Tabla 2 para la muestra mundial demuestran que no podemosnegar la hipótesis 2 con una relevanciadel $2 \%$. Resultadossimilarestambién son válidos para lassubmuestras de los países en vías de desarrollo y para los países de la OCDE.

\section{3) Corrupción y gastos en $\mathbf{F} \& \mathbf{M}$}

Una observación hecha en la sección II y muy relacionada con las H ipótesis 1 y 2, es la infrafinanciación de los gastos en 
$F \& M$. Puesto que la corrupción y los sobornos están en efecto más relacionados con las nuevas inversiones, la corrupción puede dar como resultado un gasto en $F \& M$ más bajo. Estas observa ciones conducen a la tercera hipótesis:

H ipótesis 3: Permaneciendo el resto de cosas igual, un elevado nivel de corrupción se asocia con un nivel bajo de gastos en $F \& M$.

Como ya se ha indicado anteriormente, no disponemos delos datos directos comparativos entre países sobre gastos en $F \& M{ }^{17}$. Por tanto, usamos dos sustitutos: (1) "castos sobre otros bienes y servicioss, un componente del gasto corriente, expresado como una fracción de sueldos y salarios; y (2) sueldos y salarios expresados como una fracción del gasto corriente. Estos datos se han tomado de la base de datos G FS del FM I. La lógica subyacente en el primer sustituto es obvia, puesto que, según el manual del GFS sobre las Estadísticas de Finanzas del Gobierno, los gastos en otros bienes y servicios incluyen gastos en $F \& M$. H emos expresado este gasto relativo a los sueldos y salarios para destacar potenciales intercambios entre el gasto en $F \& M$ y el gasto en sueldos y salarios. El ratio de sueldos y salarios en el gasto corriente es un sustituto razonable de los gastos en $F \& M$, porque los gobiernos a menudo tienden a conceder incrementos en sueldos, pero cortan los gastos en F\& M. D e ahí que los incrementos en sueldos y salarios puedan interpretarse como cortes en los gastos en $F \& M$.

\begin{tabular}{|c|c|c|}
\hline \multicolumn{3}{|c|}{$\begin{array}{c}\text { Tabla } 2 \\
\text { Los Efectos de la Corrupción en los Ingreos del Gobierno } \\
1980-95 \\
\text { (Como ratio de PIB, datos anuales) }\end{array}$} \\
\hline Variables independientes & (1) & (2) \\
\hline Constante & $\begin{array}{c}9,99 \\
(12,1)\end{array}$ & $\begin{array}{r}12,9 \\
(13,7)\end{array}$ \\
\hline Índice de corrupción & $\begin{array}{r}-2,51 \\
(-20,4)\end{array}$ & $\begin{array}{l}-1,71 \\
(-9,28)\end{array}$ \\
\hline PIB* real per capita & & $\begin{array}{c}3,73 \\
(5,34)\end{array}$ \\
\hline $\begin{array}{l}R^{2} \text { ajustado } \\
N \text { úmero de observaciones }\end{array}$ & $\begin{array}{l}0,272 \\
1,114\end{array}$ & $\begin{array}{l}0,28 \\
1,042\end{array}$ \\
\hline
\end{tabular}

Fuentes: FM I, Estadísticas de Finanzas del Gobierno; Tablas M undiales; Negocio Internacional; y Servicios de Riesgo Político. El índice de corrupción se toma de M AURO (1995) y la Guía Internacional de Riesgo por Países compilada por Servicios de Riesgo Político. Un valor alto del índice significa que un país tiene un elevado nivel de corrupción; t-estadísticas están entre paréntesis. La técnica de estimación es $0 \mathrm{LS}$. * Indica que el coeficiente está multiplicado por 10.000 .

\begin{tabular}{|c|c|c|c|c|c|c|}
\hline \multicolumn{7}{|c|}{ Tabla 3} \\
\hline \multicolumn{7}{|c|}{$\begin{array}{l}\text { a. Gastos en } 0 \text { tros Bienes y Servicios, } 1980-95 \\
\text { (Como ratio de sueldos y salarios, } 1980-95 \text { ) }\end{array}$} \\
\hline \multicolumn{3}{|c|}{ Mundial } & \multicolumn{2}{|c|}{ OCDE } & \multicolumn{2}{|c|}{ En vías de desarrollo } \\
\hline Variable independiente & te (1) & (2) & (1) & (2) & (1) & (2) \\
\hline Constante & $\begin{array}{l}72,9 \\
(8,15)\end{array}$ & $\begin{array}{r}97,2 \\
(9,29)\end{array}$ & $\begin{array}{c}-20,2 \\
(-0,558)\end{array}$ & $\begin{array}{c}43,4 \\
(1,19)\end{array}$ & $\begin{array}{l}84,2 \\
(7,08)\end{array}$ & $\begin{array}{l}82,3 \\
6,65\end{array}$ \\
\hline Índice de corrupción & $\begin{array}{l}-3,54 \\
(-2,69)\end{array}$ & $\begin{array}{c}4,44 \\
(2,20)\end{array}$ & $\begin{array}{c}-14 \\
(-3,53)\end{array}$ & $\begin{array}{c}5,96 \\
(1,23)\end{array}$ & $\begin{array}{l}-1,24 \\
(-0,57)\end{array}$ & $\begin{array}{c}1,43 \\
(0,60)\end{array}$ \\
\hline PIB* real per capita & & $\begin{array}{r}0,42 \\
(5,55)\end{array}$ & & $\begin{array}{c}0,81 \\
(6,99)\end{array}$ & & $\begin{array}{c}0,63 \\
(3,93)\end{array}$ \\
\hline $\begin{array}{l}R^{2} \text { ajustado } \\
N \text { úm. de observaciones }\end{array}$ & $\begin{array}{r}0,006 \\
999\end{array}$ & $\begin{array}{r}0,038 \\
927\end{array}$ & $\begin{array}{l}0,037 \\
300\end{array}$ & $\begin{array}{l}0,182 \\
273\end{array}$ & $\begin{array}{r}-0,01 \\
699\end{array}$ & $\begin{array}{r}0,021 \\
654\end{array}$ \\
\hline \multicolumn{7}{|c|}{$\begin{array}{c}\text { b. Sueldos y Salarios, } 1980-95 \\
\text { (Como ratio del gasto corriente; datos anuales) }\end{array}$} \\
\hline & \multicolumn{2}{|c|}{ Mundial } & \multicolumn{2}{|c|}{$O C D E$} & \multicolumn{2}{|c|}{ En vías de desarrollo } \\
\hline Variable independiente & te (1) & (2) & (1) & (2) & (1) & (2) \\
\hline Constante & $\begin{array}{c}47,3 \\
(41,7)\end{array}$ & $\begin{array}{c}42,2 \\
(33,2)\end{array}$ & $\begin{array}{l}34,2 \\
(12,2)\end{array}$ & $\begin{array}{c}30,8 \\
(11,3)\end{array}$ & $\begin{array}{l}39,7 \\
(26,1)\end{array}$ & $\begin{array}{c}39,7 \\
(25,2)\end{array}$ \\
\hline Índice de corrupción & $\begin{array}{c}3,1 \\
(18,5)\end{array}$ & $\begin{array}{c}1,48 \\
(6,03)\end{array}$ & $\begin{array}{l}2,17 \\
(7,02)\end{array}$ & $\begin{array}{c}0,75 \\
(2,07)\end{array}$ & $\begin{array}{c}1,22 \\
(4,43)\end{array}$ & $\begin{array}{c}1,16 \\
(3,83)\end{array}$ \\
\hline PIB* real per capita & & $\begin{array}{l}-0,84 \\
(-9,13)\end{array}$ & & $\begin{array}{l}-0,65 \\
(-7,54)\end{array}$ & & $\begin{array}{c}-0,067 \\
(-0,327)\end{array}$ \\
\hline $\begin{array}{l}R^{2} \text { ajustado } \\
\text { Núm. de observaciones }\end{array}$ & $\begin{array}{l}0,255 \\
1.000\end{array}$ & $\begin{array}{r}0,319 \\
925\end{array}$ & $\begin{array}{c}0,139 \\
300\end{array}$ & $\begin{array}{l}0,31 \\
273\end{array}$ & $\begin{array}{r}0,026 \\
700\end{array}$ & $\begin{array}{r}0,023 \\
652\end{array}$ \\
\hline
\end{tabular}

Fuentes: FMI, Estadísticas de Finanzas del Gobierno; Tablas M undiales; Negocio Internacional; y Servicios de Riesgo Político. El índice de corrupción se toma de M AURO (1995) y la Guía Internacional de Riesgo por Países compilada por los Servicios de Riesgo Político. Un valor alto del índice significa que un país tiene un alto nivel de corrupción; t-estadísticas están entre paréntesis. La técnica de estimación es 0 LS. $*$ * * indican que los coeficientes están multiplicados por 100 y 1.000, respectivamente.

Para poner a prueba la hipótesis 3 , hacemos una regresión de cada uno de los sustitutos anteriores sobre una constante y sobre un índice de corrupción y, como de costumbre en un análisis de sensibilidad, añadimos el PIB real per capita para cada regresión. Se muestran los resultados en la Tabla 3. A diferencia de las regre siones previas, presentamos los resultados para tres muestras (mundial, OCDE y páses en vías de desarrollo), puesto que hay diferencias en las tres muestras. Con respecto al primer sustituto, los 
resultados de la Tabla 3 indican que un elevado nivel de corrupción de hecho está asociado con los escasos gastos en F\& M . Sin embargo, se puede negar la hipótesis 3 con una relevancia del 1\% sólo para la muestra de los países en vías de desarrollo. Una vez que controlamos el PIB real per capita, se niega la hipótesis 3 con una relevancia del $1 \%$ para las tres muestras. Una interpretación de este descubrimiento es que el primer sustituto es un indicador ruidoso del gasto en $F \& M$.

En lo que respectaal segundo sustituto paralosgastos $F \& M$, no podemosnegar lahipótesis3 en todas lasmuestrascon unarelevancia del 1\%, tanto si controlamos como si no el PIB real per capita (Tabla 3, panel b). Los países con un elevado nivel de corrupción tienden a tener un alto ratio de sueldosy salarios sobre el gasto corriente ${ }^{18}$. Los datos son mucho más fuertes estadística y económicamente para la muestra de países en vías de desarrollo que para la muestra dela OCDE.

\section{4) Corrupción y calidad de la inversión pública}

Las inversiones en infraestructuras son a menudo desiguales y requieren de mucho capital financiero inicial. Se ha pensado durante algún tiempo que la corrupción es más frecuente en el sector de las infraestructuras(W ADE, 1982; ROSE-ACKERM AN , 1996). Las regresiones de la Tabla 1 de este documento han ofrecido datos de que la alta corrupción se asocia de hecho con una elevada inversión pública. Véase también M AURo (1997). Sin embargo, estos datos vinculan la corrupción con la cantidad de inversión, y no con la calidad. En la sección II sostuvimos que los países realizan nuevas inversiones en infraestructuras sin mantener el stock de capital existente para infraestructuras. Por tanto, cabe esperar que la calidad de las infraestructuras se deteriore y más si la corrupción conduce a recortes en el gasto en $F \& M$. Estas observaciones nos llevan a la cuarta hipótesis:

Hipótesis4: Permaneciendo igual el resto de cosas, un elevado nivel de corrupción se asocia con una escasa calidad de las infraestructuras.

Para poner a prueba esta hipótesis, hacemos una regresión de los indicadores de calidad de las infraestructuras sobre una contante, el índice de corrupción y el PIB real per capita. Los resultados se recogen en la Tabla 4 para los cinco indicadores de la calidad de las infraestructuras. La hipótesis 4 no se puede negar en los niveles de importancia usuales: los países con una elevada corrupción tienden a tener una calidad más baja de las infraestructuras. En términos de trascendencia estadística, el impacto de la corrupción es más fuerte en la calidad de las carreteras (carreteras asfaltadas en buenas condiciones), cortes de energía y ferrocarriles diesel en uso. Cuando controlamos el PIB real per capita, la corrupción cambia su signo sólo en una regresión (fallos en las telecomunicaciones) y pierde su trascendencia estadística en los niveles usuales en tres regresiones (fallos en las telecomunicacio-

\begin{tabular}{|c|c|c|c|c|c|}
\hline Variable dependiente & Constante & $\begin{array}{l}\text { Índice de } \\
\text { corrupción }\end{array}$ & $\begin{array}{l}\text { PIB* real } \\
\text { per capita }\end{array}$ & $\begin{array}{c}\mathbf{R}^{2} \\
\text { ajustado }\end{array}$ & N \\
\hline $\begin{array}{l}\text { Carreteras asfaltadas } \\
\text { en buenas condiciones }\end{array}$ & $\begin{array}{l}19,2 \\
(4,97)\end{array}$ & $\begin{array}{c}-3,84 \\
(-5,40)\end{array}$ & & 0,052 & 513 \\
\hline $\begin{array}{l}\text { Carreteras asfaltadas } \\
\text { en buenas condiciones }\end{array}$ & $\begin{array}{c}15,5 \\
(3,87)\end{array}$ & $\begin{array}{l}-2,22 \\
(-2,89)\end{array}$ & $\begin{array}{c}5,4 \\
(9,85)\end{array}$ & 0,268 & 373 \\
\hline Cortes de energía & $\begin{array}{c}18,7 \\
(27,7)\end{array}$ & $\begin{array}{c}1,1 \\
(8,69)\end{array}$ & & 0,07 & 997 \\
\hline Cortes de energía & $\begin{array}{c}18,8 \\
(32,5)\end{array}$ & $\begin{array}{c}0,95 \\
(8,17)\end{array}$ & $\begin{array}{l}-0,56 \\
(-7,07)\end{array}$ & 0,162 & 922 \\
\hline $\begin{array}{l}\text { Fallos en las } \\
\text { telecomunicaciones }\end{array}$ & $\begin{array}{c}97,6 \\
(6,93)\end{array}$ & $\begin{array}{c}4,17 \\
(1,63)\end{array}$ & & 0,007 & 241 \\
\hline $\begin{array}{l}\text { Fallos en las } \\
\text { telecomunicaciones }\end{array}$ & $\begin{array}{c}94,5 \\
(6,31)\end{array}$ & $\begin{array}{l}-0,54 \\
(-0,18)\end{array}$ & $\begin{array}{c}-9,33 \\
(-5,01)\end{array}$ & 0,127 & ¡201 \\
\hline Pérdidas de agua T & $\begin{array}{c}43,8 \\
(6,89)\end{array}$ & $\begin{array}{c}2,25 \\
(1,86)\end{array}$ & & 0,089 & 26 \\
\hline Pérdidas de agua T & $\begin{array}{c}43,6 \\
(7,19)\end{array}$ & $\begin{array}{c}1,52 \\
(1,14)\end{array}$ & $\begin{array}{l}-2,92 \\
(-1,63)\end{array}$ & 0,186 & 25 \\
\hline $\begin{array}{l}\text { Ferrocarriles diesel } \\
\text { en uso TT }\end{array}$ & $\begin{array}{c}47,1 \\
(7,45)\end{array}$ & $\begin{array}{l}-3,66 \\
(-3,80)\end{array}$ & & 0,17 & 67 \\
\hline $\begin{array}{l}\text { Ferrocarriles diesel } \\
\text { en uso TT }\end{array}$ & $\begin{array}{l}59,4 \\
(8,62)\end{array}$ & $\begin{array}{l}-0,58 \\
(-0,46)\end{array}$ & $\begin{array}{c}1,37 \\
(3,39)\end{array}$ & 0,285 & 67 \\
\hline
\end{tabular}

Fuentes: FM I, Estadísticas de Finanzas del Gobierno; Tablas M undiales; N egocio Internacional; y Servicios de Riesgo Político. El índice de corrupción se toma de M AURo (1995) y la Guía Internacional de Riesgo por Países compilada por los Servicios de Riesgo Político. Un valor alto del índice significa que un país tiene un elevado nivel decorrupción; t-estadísticas están entre paréntesis. La técnica de estimación es 0 LS. T y T T denotan las medias de los datos en los períodos 1980-89 y 1990-95, respectivamente.

* Indica que el coeficiente está multiplicado por 10.000 .

nes, pérdidas de agua y ferrocarriles diesel en uso). El ajuste de cada regresión mejora, como se puede juzgar por la R al cuadrado ajustado, cuando añadimos el PIB real per capita. Además, el PIB real per capita en cada regresión tiene el signo adecuado: los páses con un PIB real per capita mayor tienden a tener una me jor calidad de las infraestructuras. Una implicación importante de los resultados de la Tabla 4 es que los costes de la corrupción también se deberían medir en términos del deterioro de la calidad de las infraestructuras existentes. Estos costes pueden ser muy altos en términos de su gran impacto en el crecimiento. 


\begin{tabular}{|c|c|c|c|c|}
\hline Los Efectos de la Corrt & $\begin{array}{r}\text { Tab| } \\
\text { pción En } \\
1980\end{array}$ & Calio & elas & reteras, \\
\hline $\begin{array}{l}\text { Variable dependien } \\
\text { condiciones como p }\end{array}$ & $\begin{array}{l}\text { te: carre } \\
\text { orcentaj } \\
\text { asfalt: }\end{array}$ & $\begin{array}{l}\text { as affa } \\
\text { del tota } \\
\text { as }\end{array}$ & $\begin{array}{l}\text { sen } \\
\text { las } C\end{array}$ & $\begin{array}{l}\text { nas } \\
\text { eras }\end{array}$ \\
\hline & (D atos al & & & \\
\hline Variables independientes & (1) & (2) & (3) & (4) \\
\hline Constante & $\begin{array}{c}-1,03 \\
(-0,150)\end{array}$ & $\begin{array}{c}7,55 \\
(1,01)\end{array}$ & $\begin{array}{c}1,83 \\
(0,193)\end{array}$ & $\begin{array}{c}19,6 \\
(1,82)\end{array}$ \\
\hline Índice de corrupción & $\begin{array}{c}-7 \\
(-8,68)\end{array}$ & $\begin{array}{l}-2,56 \\
(-2,20)\end{array}$ & $\begin{array}{c}-6,51 \\
(-4,74)\end{array}$ & $\begin{array}{c}-0,32 \\
(-0,17)\end{array}$ \\
\hline Ratio inversión pública-PIB & $\begin{array}{c}2,03 \\
(2,65)\end{array}$ & $\begin{array}{c}3,09 \\
(4,00)\end{array}$ & $\begin{array}{c}1,15 \\
(0,53)\end{array}$ & $\begin{array}{l}-0,2 \\
(0,10)\end{array}$ \\
\hline $\begin{array}{l}\text { Ratio inversión pública-PIB } \\
\text { por el Índice de corrupción }\end{array}$ & & & $\begin{array}{l}-0,16 \\
(-0,44)\end{array}$ & $\begin{array}{l}-0,58 \\
(-1,56)\end{array}$ \\
\hline PIB real per capita * & & $\begin{array}{c}0,24 \\
(6,38)\end{array}$ & & $\begin{array}{c}0,25 \\
(6,57)\end{array}$ \\
\hline $\begin{array}{l}\mathrm{R}^{2} \text { ajustado } \\
\mathrm{N} \text { úmero de observaciones }\end{array}$ & $\begin{array}{l}0,186 \\
322\end{array}$ & $\begin{array}{l}0,326 \\
269\end{array}$ & $\begin{array}{l}0,184 \\
322\end{array}$ & $\begin{array}{c}0,329 \\
269\end{array}$ \\
\hline
\end{tabular}

Fuentes: FMI, Estadísticas de Finanzas del Gobierno; Tablas M undiales; Negocio Internacional; y Servicios de Riesgo Político. El índice de corrupción se toma de M Auro (1995) y la Guía Internacional de Riesgo por Países compilada por los Servicios de Riesgo Político. Un valor alto del índice significa que un país tiene un elevado nivel decorrupción; t-estadísticas están entre paréntesis. La técnica de estimación es $0 \mathrm{LS}$. * Indica que el coeficiente está multiplicado por 100.

¿Reduce la corrupción la calidad de lasinfraestructurasa través de la inversión pública?

Para contestar a la cuestión anterior, llevamos a cabo un test más riguroso de la hipótesis 4 en cuanto a la calidad de las carre teras ${ }^{19}$. H acemos una regresión de las carreteras asfaltadas en bue nas condiciones sobre una constante, el PIB real per capita, el índice de corrupción (p.e., la misma regresión que en la Tabla 4) y dos variables adicionales: el ratio inversión pública-PIB y su interacción con el índice de corrupción. Los resultados se recogen en la Tabla 5. Las columnas (1) y (2) muestran que, incluso cuando controlamos la inversión pública, no podemos negar la hipótesis 4 con una relevancia del $1 \%$. La regresión de la columna (3) muestra que la corrupción todavía es significativa ante la presencia de la variable de la interacción. Si la corrupción reduce la calidad de las carreteras a través de la inversión pública, debe ríamos descubrir que la corrupción pierde su importancia cuando la variable de interacción se añade a la regresión, dada la pre sencia del ratio inversión pública-PIB y el PIB per capita real. La comparación entre las columnas (4) y (2) - con y sin el término de la interacción respectivamente- demuestra que esto es lo que ocurre. Además, el término de la interacción estadísticamente significativo en la columna 4 demuestra que el impacto de la corrupción en la calidad de las carreteras depende de la inversión pública. El signo negativo en el término de la interacción sugiere que cuanto mayor es la inversión pública, mayor es el impacto negativo de la corrupción en la calidad de las carreteras. Estos datos adicionales son coherentes con el descubrimiento de la Tabla 1 de que una elevada corrupción está asociada de hecho con una mayor inversión pública.

¿Una mayor corrupción reduce la productividad de la inversión pública?

Supongamos que medimos la productividad de la inversión pública en función de las mejoras en la calidad de las carreteras por dólar de inversión pública. La regresión en la columna (4) de la Tabla 5 demuestra que el impacto de la inversión en la calidad de las carreteras depende de la existencia de la corrupción. Espe cíficamente, el signo negativo en el término de interacción de muestra que una mayor corrupción puede reducir la productividad de la inversión pública.

\section{Conclusiones}

$H$ ay muchos canales a través de los cuales una elevada corrupción reduce el crecimiento económico. M AURo (1995) ofrece datos y resume algunos de estos argumentos. Los nuevos datos presentados en este trabajo apoyan cuatro argumentos adicionales.

Primero, la corrupción puede reducir el crecimiento, incre mentando la inversión pública al tiempo que reduce su productivi$\mathrm{dad}^{7}$. Este hall azgo es coherente con las típicas regresiones comparativas entre países pequeños. Por ejemplo, D evarajan, Swaroop y Zou (1996) han descubierto que cuanto mayor es la inversión pública menor es el crecimiento, dados otros determinantes de crecimiento y T ANzI (1994) descubrió que la relación entre el crecimiento y la inversión se ve muy influenciada por la inclusión de un par de países.

Segundo, la corrupción puede reducir el crecimiento, incre mentando la inversión pública que no va acompañada por un gasto corriente recurrente, p.e., unos gastos adecuados no salariales en $F \& M$. Nuestros datos demuestran que una mayor corrupción va asociada a un mayor gasto total en los sueldos y salarios. Los sueldos y salarios son un componente importante del consumo del gobierno y se ha demostrado que el mayor consumo del gobierno está relacionado de forma inequívoca con un menor crecimiento (COM m AN D ER et al., 1997; BARRO, 1996; BARRO y SAla-I-M ARTIN, 1995).

Tercero, la corrupción puede reducir el crecimiento, reduciendo la calidad de las infraestructuras existentes. Una infra- 
estructura que se va deteriorando aumenta el coste de hacer negocios, tanto para el gobierno como para el sector privado (p.e., congestión, retrasos, averías de maquinaria, etc.) y, por tanto, conduce a una producción y a un crecimiento más bajos. La importancia de la infraestructuras en el crecimiento ha quedado demostrada en muchas regresiones de crecimiento comparativas entre países (CANNIng y FAY, 1993; EASTERLY y LeVINE, 1996; HuLTEN, 1996).

Finalmente, la corrupción puede reducir el crecimiento disminuyendo los ingresos del gobierno que se necesitan para financiar el gasto productivo.

La implicación de este trabajo es que los economistas debe rían ser más comedidos en sus elogios sobre el elevado gasto en inversión del sector público y de las reglas, como la regla de oro, especialmente en los países donde la corrupción y, concretamente el elevado nivel de corrupción, es un problema.

Este documento se ha centrado en el problema de la corrupción y no en las soluciones. En la medida en que la corrupción hace referencia a las actividades de las empresas extranjeras, la OCDE está intentando en la actualidad inducir a los países industriales a: (a) hacer que los pagos de los sobornos a los funcionarios extranjeros no sean fiscalmente deducibles; y (b) tipificar como delito el pago de sobornos. H asta ahora los ministros que representan a los países de la OCDE han aceptado estas recomendaciones, pero los órganos legislativos de aquellos países de ben actuar ahora. La propuesta de la OCDE, sin embargo, no afectaría a los proyectos de inversión pública en los países que no pertenecen a la OCDE llevados a cabo por los contratistas nacionales o por contratistas de países queno pertenecen a la 0 CDE ${ }^{21}$.
Artículo traducido por Gema Sán chez Rontomé.

* Fondo Monetario Internacional.

${ }^{1}$ Este documento se presentó en el 53 Congreso del Instituto Internacional de Finanzas Públicas, Kyoto, Japón, 25-28 de agosto de 1997. Los autores querrían agradecer a Tarja PAPAVASSILIO u su ayuda con el ordenador.

${ }^{2} \mathrm{TI}$ es una organización no gubernamental, con sede en Berlín que hace un seguimiento de las tendencias de corrupción en todo el mundo y que tiene como objetivo la eliminación de la corrupción.

${ }^{3}$ La literatura distingue entre la corrupción en pequeña escala o corrupción burocrática y la corrupción «a gran escala» o corrupción política.

${ }^{4} \mathrm{~L}$ a regla simplemente establece que el gasto corriente sólo debe equilibrarse con los ingresos ordinarios: un país puede tener un déficit fiscal igual al gasto de capital neto del gobierno.

${ }^{5}$ Pensiones, pagos de interés de las deudas, salarios, subsidios, etc.

${ }^{6}$ Los políticos concretos no tienen generalmente el poder para cambiar las pensiones, salarios o subsidios de individuos concretos.

${ }^{7}$ A menudo, la comisión es un eufemismo de lo que en esencia es un soborno.

${ }^{8}$ D e hecho, en muchos casos el acto de soborno puede no comenzar con las empresas, sino con los funcionarios que controlan las decisiones. Las empresas extranjeras informan de que en al gunos países es imposible conseguir un contrato del gobierno sin pagar un soborno.

${ }^{9}$ Para un estudio útil sobre la corrupción en la inversión pública, véase Patrick MeAgher (1997).

${ }^{10}$ Esta segunda opción puede ser menos atractiva para la empresa si teme que el funcionario pueda exigir pagos adicionales cuando se realizan modificaciones que suponen un incremento del gasto o si teme que el funcionario pueda dejar de tener poder para influir en el proceso. En los países en donde los mismos individuos permanecen en el poder durante mucho tiempo, la estrategia de dar una oferta inicial baja seguida de ajustes a lo largo del período en el que se ejecuta el proyecto es una estrategia común.

${ }^{11}$ Esto ha ocurrido frecuentemente en la construcción de carreteras en donde el espesor dela base de la carretera se puede reducir mucho. También ha ocurrido en la construcción de puentes y edificios que, a veces, se han derrumbado, causando la pérdida de vidas y costes económicos.

${ }^{12}$ En Italia antes de tangentopoli, los contratados para evaluar los proyectos a menudo se encontraban con que se les ignoraba totalmente.

${ }^{13}$ Esto puede formar parte de la razón por la cual observamos ratios de capitaloutput extremadamente altos en algunos países.

${ }^{14}$ Los estudios del Banco M undial indican que en muchos países las infraestructuras públicas, incluidas carreteras, centrales eléctricas, canales de riego, con frecuencia sólo se pueden usar en una parte de su capacidad total.

${ }^{15}$ Los dos índices tienen una alta correlación con un coeficiente de correlación de 0,81. También se dispone de otros índices, incluido el publicado por Transparencia Internacional (Transparency International). Estos índices también tienen una alta correlación.

${ }^{16}$ O bsérvese que la corrupción reduce la inversión agregada (M AURO, 1995) que es la suma de la inversión de capital público y privado. Por tanto, la corrupción debe reducir la inversión en capital privado más de lo que incrementa la inversión de capital público.

${ }_{17}$ De manera ideal, queremos incrementos en los gastos en $F \& M$. Esto requiere conocer los denominados coeficientes «r» y los gastos reales en $F \& M$. El coeficiente $r$ es el ratio entre las exigencias de gasto neto recurrente en el coste total de la inversión de un proyecto; véase H ELLER (1991).

${ }^{18}$ Esto no significa que el nivel desalarios en los países corruptos es mayor. D ehecho, un estudio reciente ha descubierto que hay una relación negativa entre los niveles salarialesen el sector público y la corrupción. Véase V AN RIJCKEGH EM yW EDER (1997). ${ }^{19}$ Los resultados son similares a otras mediciones de la calidad de las infraestructuras. 20 Por favor, nóteseque, debido a quela corrupción reducelos ingresos fiscales, es probable que el incremento relativo de la inversión pública (p.e. su cuota del presupuesto total del gobierno) sea mayor que el incremento absoluto en la inversión pública. ${ }^{21}$ Para un estudio sobre los pasos a dar para reducir la corrupción, véase Tanz। (1997). 
BARRO, Robert J. (1996), «D eterminants of Economic Growth: A Cross-Country Empirical Sudy», N BER Working Paper, no. 5698.

BarRo, Robert J. y X. SALA-I-M ARTIn (1995), Economic Growth, Mc Graw Hill, Nueva York.

Cann ING, David y M arianne Fay (1993), «T he Effect of Transportation N etworks on Economic Growth», mimeo, Universidad de Columbia.

Commander, Simon, Hamid R. Davoodi, Une J. Lee (1997), «The Causes of Government and the Consequences for Growth and Well-Being", World Bank Policy Research Paper N 0. 1785.

Devarajan, Shantayanan, VinayaSwarro p y Heng-fu Zou (1996), «TheComposition of Public Expenditure and Economic Growth», Journal of M onetary Economics, 37, pp. 313-344.

EASTERLy, William y R. LeVIne (1996), «Africa's Growth Tragedy», mimeo, Banco Mundial.

Heller, Peter S. (1991), «0 perations and M aintenance», Public Expenditure H andbook, editado por KeYoung Chu y Richard Hemming, FM I.

H ULt En, CharlesR. (1996), «nfraestructureand Economic D evelopment: 0 neM ore Unto the Beach», mimeo, Banco M undial y U niversidad de M aryland, College Park.

Knack, Stephen y Philip Keefer (1995), «nstitucions and Economic Performance: Cross-Country Tests Using Alternative Institutional M easuress, Economics and Politics, Vol. 7, No 3, pp. 207-227.

KlitgaARD, Robert (1988), Controlling Corruption, Berkeley, Universidad de California.

M AURO, Paolo (1997), «The Effects of Corruption on Growth, Investment, and Government Expenditure: A C ross Country Analysis» en Corruption and the G lobal Economy, ed. por Kimberly Ann Elliott, Washington: Institute for International Economics.

M AURO, Paolo (1995). «Corruption and G rowth», Q uarterly Journal of Economics, CX., no. 3 (Agosto), pp. 681-712.
Meagher, Patrick (1997), «Combating Corruption in Africa; Institutional Challenges and Response», documento presentado en el seminario del FM I sobre Luchando contra la Corrupción en la G estión Económica y Financiera, Lisboa, 1921 de M ayo.

O LSON, M ancur (1996), «Big Bills Left on the Sidewalk: Why Some Nations are rich and 0 thers Poor,》 Journal of Economic Perspectives, 10, 2, pp. 3-24.

Pritchett, Lant (1996), «M ind Your P's and Q 's: The Cost of Public Investment is not the Value of Public Capital Stock», Policy Ressearch Working Paper, no 1660, Banco M undial, Washington D.C.

RoSE-ACKERM AN, Susan (1996), «W hen is Corruption H armful?» Background paper for the 1997 World Development Report.

SvensSON, Jacob (1996), «Foreign Aid and Rent-Seeking», Banco M undial, División de M acroeconomía y Crecimiento, mimeo.

T AnzI, Vito (1991), PublicFinancein D eveloping Countries, Aldershot: Edward Elgar.

Tanzl, Vito (1994), «The IMF and Tax Reform», en Tax Policy and Planning in D eveloping Countries, editado por Amaresh Bagchi y N icholas Stern, Delhi: 0 xford University Press.

Tanzl, Vito (1995), «Corruption, Government Activities, and M arkets» en The Economics of Organized Crime, editado por Gianluca Fiorentini y Sam Peltzman, Cambridge: Cambridge University Press.

TAnzI, Vito (1997), «Corruption, G overnment Activities and Policy Instruments: A Brief Review of the M ain Issues» (mimeo: mayo 1997).

Van RIJCKEghem, Caroliney Beatrice W eder (1997), «Corruption and the rate of Temptation: Do Law Wages in the Civil Service Cause Corruption?» D ocumento de Trabajo del FMI.

W ADE, Robert (1982), «The System of Administrative and Political Corruption: Canal Irrigation in South India», Journal of D evelopment Studies, 18, pp. 287-328.

W El, Shang-Jin, «H ow Taxing is Corruption on International Investors», Series de D ocumentos de Trabajo de N BER, D ocumento de Trabajo 6030. 